\title{
REG Y knockdown suppresses proliferation by inducing apoptosis and cell cycle arrest in osteosarcoma
}

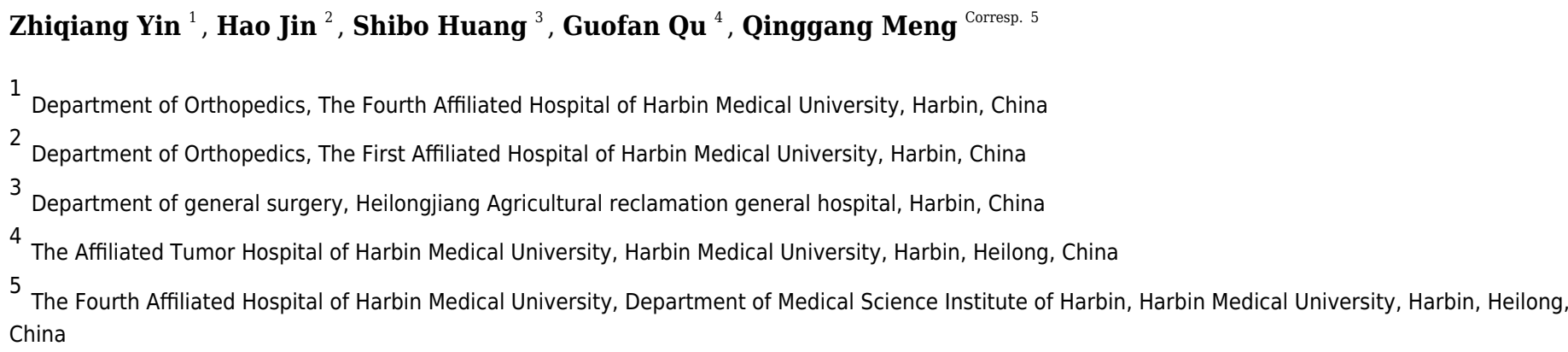

Background: Osteosarcoma (OS) is the most common malignant bone tumor with high mortality in children and adolescents. REG $\gamma$ is overexpressed and plays oncogenic roles in various types of human cancers. However, the expression and potential roles of REG $\gamma$ in osteosarcoma are elusive. This study aims at exploring possible biological functions of REG $\gamma$ in the pathogenesis of osteosarcoma and its underlying mechanism.

Methods: Quantitative reverse transcription-polymerase chain reaction (qRT-PCR), western blotting and immunohistochemistry (IHC) were performed to detect the expression levels of REG $\gamma$ in OS tissues and cell lines. Then, the effects of REG $\gamma$ expression on OS cell proliferation in vitro were analyzed by Cell Counting Kit-8 (CCK-8), ethylene deoxyuridine (EdU), colony formation, flow cytometry. The protein levels of apoptosis and cell-cycle related proteins were evaluated using western blotting.

Results: In present study, we found for the first time that REG $\gamma$ is overexpressed in osteosarcoma tissues and cell lines and knockdown of REG $\gamma$ significantly inhibits cell proliferation and induces apoptosis and cell cycle arrest in osteosarcoma cells. Furthermore, we observed that p21, caspase-3 and cleaved caspase-3 are increased while the expression of cycinD1 and bcl-2 are decreased after REG $\gamma$ depletion in osteosarcoma cells. In conclusion, REG Y may involve in proliferation of osteosarcoma and serve as a novel therapeutic target in patients with osteosarcoma. 
1

2

3 Zhiqiang Yin ${ }^{1,6}$, Hao Jin ${ }^{2}$, Shibo Huang ${ }^{3}$, Guofan Qu ${ }^{4, *}$ and Qinggang Meng ${ }^{1,5 *}$

$4{ }^{1}$ Department of Orthopedics, The Fourth Affiliated Hospital of Harbin Medical University,

5 Harbin, China;

$6{ }^{2}$ Department of Orthopedics, The First Affiliated Hospital of Harbin Medical University,

7 Harbin, China;

$8{ }^{3}$ Department of general surgery, Heilongjiang Agricultural reclamation general hospital, Harbin,

9 China;

$10{ }^{4}$ Department of Orthopedics, The Affiliated Tumor Hospital of Harbin Medical University,

11 Harbin Medical University, Harbin, China.

$12{ }^{5}$ Department of Medical Science Institute of Harbin, Harbin, China.

$13{ }^{6}$ The Key Laboratory of Myocardial Ischemia, Chinese Ministry of Education, Harbin, China.

$14 *$ Corresponding author:

15 Qing-Gang Meng*

16 The Fourth Affiliated Hospital of Harbin Medical University, Department of Medical Science

17 Institute of Harbin, Harbin Medical University, Harbin, China.

18 Email: mqg138456@163.com

19 Tel.: +86045184883003

20 Guofan $\mathrm{Qu}^{*}$ 
21 The Affiliated Tumor Hospital of Harbin Medical University, Harbin Medical University,

22 Harbin, China.

23 Email: guofanqu doctor@,163.com

\section{Abstract:}

Background: Osteosarcoma (OS) is the most common malignant bone tumor with high

mortality in children and adolescents. REG $\gamma$ is overexpressed and plays oncogenic roles in

various types of human cancers. However, the expression and potential roles of REG $\gamma$ in

osteosarcoma are elusive. This study aims at exploring possible biological functions of REG $\gamma$ in the pathogenesis of osteosarcoma and its underlying mechanism.

Methods: Quantitative reverse transcription-polymerase chain reaction (qRT-PCR), western blotting and immunohistochemistry (IHC) were performed to detect the expression levels of

REG $\gamma$ in OS tissues and cell lines. Then, the effects of REG $\gamma$ expression on OS cell

proliferation in vitro were analyzed by Cell Counting Kit-8 (CCK-8), ethylene deoxyuridine

35 (EdU), colony formation, flow cytometry. The protein levels of apoptosis and cell-cycle related proteins were evaluated using western blotting.

Results: In present study, we found for the first time that REG $\gamma$ is overexpressed in osteosarcoma tissues and cell lines and knockdown of REG $\gamma$ significantly inhibits cell proliferation and induces apoptosis and cell cycle arrest in osteosarcoma cells. Furthermore, we

40 observed that $\mathrm{p} 21$, caspase- 3 and cleaved caspase- 3 are increased while the expression of

41 cycinD1 and bcl-2 are decreased after REG $\gamma$ depletion in osteosarcoma cells. In conclusion, 
42

43

REG $\gamma$ may involve in proliferation of osteosarcoma and serve as a novel therapeutic target in patients with osteosarcoma.

\section{Key words: Osteosarcoma; Proliferation; REG $\gamma$}

\section{Introduction:}

Osteosarcoma (OS) is the most common primary malignant bone cancer affecting children and adolescents(Botter et al. 2014). Although OS only accounts for less than $0.2 \%$ of all cancers, mortality rate of OS is up to $50 \%$ in children(Siegel et al. 2016). Despite rapid development in treatment strategies, prognosis of patients with OS has shown no significant improvement in nearly 20 years(Kempf-Bielack et al. 2005; Marko et al. 2016). As a result, there is an unmet need to identify novel molecules involved in the tumorigenesis of OS, which will be beneficial for treatment of patients with OS.

REG $\gamma$, also known as PSME3, PA28g or Ki antigen, is a member of the 11S family of proteasome activator and plays crucial roles in an ubiquitin- and ATP-independent nonlysosomal intracellular protein degradation(Ma Chu-Ping et al. 1993). Previous researches showed that REG $\gamma$-knockout mice and cells display growth retardation, reduced cell proliferation and increased apoptosis(Li et al. 2013). Moreover, REG $\gamma$ can promote the degradation of multiple tumor suppressor proteins (Chen et al. 2007; Li et al. 2015b; Li et al. 2006; Wang et al. 2015) and maintain stability of centrosome and chromosomal(Zannini et al. 2008). Additionally, growing evidence have confirm that REG $\gamma$ is overexpressed in multiple types of cancers, such as skin cancer ( $\mathrm{Li}$ et al. 2015a), breast cancer(Yi et al. 2017), renal cell cancer(Chen et al. 2018) and thyroid carcinoma(Zhang et al. 2012). However, the expression and 
63 biological functions of REG $\gamma$ in OS have never been elucidated.

64 This study aimed to elucidate the expression and biological functions of REG $\gamma$ in OS. We

65 found that REG $\gamma$ is overexpressed in OS tissues and cell lines compared with the adjacent

66 normal tissues and the normal osteoblast hFOB1.19 cell, respectively. In addition, we found that

67 knockdown of REG $\gamma$ significantly inhibited proliferation and induced apoptosis and cell cycle

68 arrest in MG-63 and SaoS-2 cell lines. Moreover, we also observed that multiple apoptosis and

69 cell cycle related proteins expression were altered in REG $\gamma$-silenced osteosarcoma cells. Our

70 results demonstrated that REG $\gamma$ plays an oncogenic role in osteosarcoma and may be a

71 molecular target in the treatment of patients with OS.

72 Materials and methods:

\section{Clinical tissue samples}

74 A total of ten OS tissues and ten adjacent normal tissues were obtained from primary

75 osteosarcoma patients who underwent operative treatment at the Third Affiliated Hospital of

76 Harbin Medical University from June 2018 to December 2018. Nine of the patients received

77 preoperative chemotherapy. Partial tissue specimens were snap-frozen immediately in liquid

78 nitrogen and stored at $-80{ }^{\circ} \mathrm{C}$ until use. The other samples were fixed with $4 \%$ paraformaldehyde

79 and then immunohistochemically stained. This study was approved by the Ethics Committees of

80 the Third Affiliated Hospital of Harbin Medical University and written informed consent was

81 obtained from each patient.

82 Cell culture

83 Human OS cell lines MG-63 and SaoS-2 were cultured in RPMI-1640 medium. The normal 
osteoblast hFOB1.19 cell was cultured in F-12 medium. All media were supplemented with 10\% fetal bovine serum, $100 \mathrm{U} / \mathrm{ml}$ penicillin, and $100 \mathrm{mg} / \mathrm{ml}$ streptomycin. Cells were cultured in a humidified incubator at $37^{\circ} \mathrm{C}$ with $5 \% \mathrm{CO}$. The two OS cell lines and hFOB1.19 were obtained from Cell Bank of the Chinese Academy of Sciences (Shanghai, China).

\section{Immunohistochemistry}

Tissue samples were fixed with $4 \%$ paraformaldehyde, dehydrated by a gradient series of ethanol, and then embedded in paraffin. The 4- $\mu \mathrm{m}$ sections were deparaffinized, rehydrated, and then stained with hematoxylin and eosin (H\&E). Next, the tissue sections were subjected to antigen retrieval, be blocked with goat serum and incubated with a primary antibody at $4{ }^{\circ} \mathrm{C}$ overnight. Subsequently, the sections were incubated with a goat anti-rabbit secondary antibody for $20 \mathrm{~min}$ at room temperature and then for $30 \mathrm{~min}$ with Streptavidin-HRP peroxidase. Diaminobenzidine (DAB)-H2O2 was used as a substrate for the peroxidase enzyme. Then, the sections were stained with hematoxylin and dehydration. The primary antibody (REG $\gamma$ rabbit polyclonal antibody) used for IHC analysis was purchased from Proteintech (catalog number: 14707-1-AP).

\section{Transient transfection}

Three small interfering RNAs specifically targeting human REG $\gamma$ (siRNA-REG $\gamma$ ) and a nonspecific negative control oligo (siRNA-NC) were purchased from GenePharma (Shanghai, China). The sequences of siRNA-REG $\gamma$ and siRNA-NC were shown in Table 1. Cell forward transfections were performed using Lipofectamine 2000 (Invitrogen; Thermo Fisher Scientific, Inc. USA) according to the manufacturer's instructions. Briefly, $5 \mu 1$ Lipofectamine 2000 and $5 \mu 1$ 
105

106

siRNA $(20 \mu \mathrm{M})$ were used in 6-well plate. Serum-free media (optiMEM) was used to dilute the siRNA and transfection reagents. Transfection efficiency of siRNA-REG $\gamma-1$ and siRNA-REG $\gamma-2$ was more than 50 percent, so they were used in following researches. Total RNA and total protein were extracted after $48 \mathrm{~h}$ and $72 \mathrm{~h}$ of transfection, respectively.

\section{Western blot}

Total protein was extracted from tissue samples or cultured cells by using a cold RIPA buffer (Beyotime Biotechnology, Shanghai, China) with protease inhibitor cocktail (Sigma-Aldrich, USA) on ice. Protein concentration was quantified by bicinchoninic acid (BCA) protein assay kit (Beyotime Biotechnology, Shanghai, China). Equal amount of protein was separated by $12 \%$ SDS-PAGE and transferred onto a polyvinylidene fluoride membrane (PVDF, Millipore, MA, USA (Catalog number: IPVH00010 and ISEQ00010)). Membranes were blocked with 5\% fatfree milk in PBS for $2 \mathrm{~h}$ at room temperature and then incubated with a primary antibody at $4{ }^{\circ} \mathrm{C}$ overnight. After washed with TBST, membranes were incubated with an HRP-labeled secondary antibody for $1 \mathrm{~h}$ at $4{ }^{\circ} \mathrm{C}$. Finally, membranes were washed three times, chemiluminescent reagent (BeyoECL Plus, Beyotime, Shanghai, China (Catalog number: P0018)) was added on the membranes and the specific signals were visualized by a Tanon Chemiluminescence Imaging System (Shanghai, China). The intensity was determined using ImageJ software. The expression level of every protein was independently detected. The primary antibodies used for WB were purchased from Proteintech (REG $\gamma$ (catalog number:14907-1-AP), $\beta$ - actin (catalog number:20536-1-AP)) and Cell Signaling Technology (p21 (catalog number:2947), bcl-2 (catalog number:2872), caspase-3 (catalog number:9662), cleaved caspase-3 (catalog 
126

127

128

129

130

131

132

133

number:9661), cyclin D1 (catalog number:2922)).

\section{RNA isolation and qRT-PCR analysis}

Total RNA was extracted from the OS tissue and cultured cells using TRIzol reagent (Invitrogen, CA, USA) and cDNA synthesis kit (Toyobo, Kyoto, Japan) was used in generating cDNA according to the manufacturer's protocol. Quantitative reverse transcription-polymerase chain reaction (qRT-PCR) was performed using SYBR Green PCR kit (Toyobo, Kyoto, Japan) with an CFX96 Touch real time machine (Bio-Rad, USA) according to manufacturers' instructions. DNA amplification was programmed for an initial $95^{\circ} \mathrm{C}$ for $60 \mathrm{~s}$, followed by 40 cycles of $95^{\circ} \mathrm{C}$ $15 \mathrm{~s}, 60^{\circ} \mathrm{C} 15 \mathrm{~s}$ and $72^{\circ} \mathrm{C} 45 \mathrm{~s}$, and ended with $72^{\circ} \mathrm{C} 5 \mathrm{~min}$. The relative expression level of target gene mRNA normalized to $\beta$-actin was determined by the $2-\Delta \Delta \mathrm{Ct}$ method. All the primers used in this study were showed in Table1.

\section{CCK-8 assay}

Cells transfected with siRNA for $24 \mathrm{~h}$ were seeded in a 96-well plate $(100 \mu 1,2000$ cells per well). The viability of transfected MG-63 and SaoS-2 cells were determined by a Cell Counting Kit (CCK-8, Dojindo, Tokyo, Japan) at 24, 48 and $72 \mathrm{~h}$ and $10 \mu \mathrm{CCK}-8$ solution was added to each well before measurement. After $2 \mathrm{~h}$ of incubation, the optical density at $450 \mathrm{~nm}$ was detected by an ultraviolet spectrophotometer (Bio-Rad, Hercules, CA, USA).

\section{Colony formation assay}

After being transfected for $24 \mathrm{~h}$, the cells were plated into 6-well plates at a density of $1.5 \times$ $10^{3} /$ well and cultured for approximately five days until visible colonies formed. Cells were washed twice with cold PBS, fixed with 4\% paraformaldehyde and then stained with $0.1 \%$ 
147 crystal violet (Beyotime Biotechnology, Shanghai, China). The number of colony formation was

148 counted and photographed with a digital camera.

149 EdU assay

150 Cell proliferation was measured using the Cell-Light ${ }^{\mathrm{TM}}$ EdU Apollo ${ }^{\circledR} 488$ In Vitro

151 Imaging kit (Ribobio, Guangzhou, China (catalog number:C10310-1)) according to the

152 manufacturer's instructions. Briefly, MG-63 and SaoS-2 cells transfected with siRNA for $24 \mathrm{~h}$

153 were seeded into 96-well plates at a density of $6 \times 10^{3} /$ well. After 24 hours, cells were incubated

154 with $50 \mu \mathrm{M}$ EdU for $2 \mathrm{~h}$. Then, the cells were fixed with $4 \%$ paraformaldehyde and the cell nuclei

155 were stained with Hoechst 33342. Subsequently, the EdU-positive cells were captured and

156 counted with a fluorescent microscope.

\section{Apoptosis assay and cell cycle analysis}

To analyze cells apoptosis rate, FITC-Annexin V Apoptosis Detection Kit (BD Biosciences, San

Jose, CA (catalog number:556547)) was used according to the manufacturer's instructions. After

being transfected for $48 \mathrm{~h}$, cultured cells were collected, washed twice with cold PBS and

propidium iodide (PI) in the dark for $15 \mathrm{~min}$ at room temperature. For the cell cycle analysis,

163 cells transfected with siRNA for $48 \mathrm{~h}$ were harvested, washed twice with precooled PBS, and

164 fixed in $70 \%$ precooled ethanol at $4{ }^{\circ} \mathrm{C}$ overnight. Then, the cells were washed with precooled

PBS and resuspended in $500 \mu$ solution containing PI $(50 \mu \mathrm{g} / \mathrm{ml})$ and RNase A $(50 \mu \mathrm{g} / \mathrm{ml})$ in

166 the dark at room temperature for $20 \mathrm{~min}$. All the flow experiments were performed using BD

167 FACS Calibur (Beckman Coulter, CA, USA). In addition, FlowJo_V10 software was used in cell 
168

169

170

171

172

173

174

175

176

177

178

179

apoptosis analysis and ModFit LT software was used in cell cycle analysis.

\section{Statistical analysis}

Data were presented as the mean \pm SD and analyzed with Student's $t$-test or one-way ANOVA

by GraphPad Prism7.0. All tests were two-sided, and a $P$-value of $<0.05$ was considered statistically significant. All experiments were independently performed three times.

\section{Results:}

1. REG $\gamma$ is upregulated in OS tissue and cell lines at both protein and mRNA levels

The expression of REG $\gamma$ was examined in osteosarcoma tissues and adjacent normal tissues

by using IHC, WB and qRT-PCR analysis. Clinical characteristics of included OS patients were shown in Table 2. Results demonstrated that REG $\gamma$ expression was significantly overexpressed in OS tissues compared with adjacent normal tissues (Figure 1A, B, C, D). To further confirm the upregulated expression of REG $\gamma$ in OS, western blot and RT-PCR analysis were performed in OS cell lines (MG-63 and SaoS-2) and human normal osteoblast (hFOB1.19) (Figure 1E, F).

Meanwhile, bioinformatic results from the Oncomine open cancer microarray database

(https://www.oncomine.org/) also shown that REG $\gamma$ mRNA levels were higher in sarcoma than in normal tissues $(p<0.01)$ (Figure $1 \mathrm{G}, \mathrm{H}, \mathrm{I})$.

2. SiRNAs targeting REG $\gamma$ reduce the expression of REG $\gamma$ at mRNA and protein level in OS cells

To reduce the expression of REG $\gamma$ and avoid off-target phenomenon, the cells were transfected with three different siRNAs targeting REG $\gamma$ and with Si-NC as control. The qRTPCR analysis showed significantly decreased levels of REG $\gamma$ mRNA in Si-REG $\gamma-1$ and Si- 
189

190

191

192

193

REG $\gamma$-2 groups compared to Si-NC group $(p<0.05)$ (Figure 2A, B). Consistently, Si-REG $\gamma-1$

and Si- REG $\gamma$-2 also markedly inhibited the REG $\gamma$ expression at protein levels as shown as in western blot analysis (Figure 2C, D). Conclusively, Si-REG $\gamma$-1 and Si-REG $\gamma$-2 efficiently downregulated REG $\gamma$ expression.

3. REG $\gamma$ knockdown inhibits proliferation in MG-63 and SaoS-2 cells.

To confirm REG $\gamma$ biological functions in osteosarcoma, we performed a series of functional assays in cells after transfection. Compared to Si-NC, siRNA-REG $\gamma-1$ and siRNA-REG $\gamma-2$ were able to effectively suppressed OS cells growth determined by CCK-8 $(p<0.05)$ (Figure $3 \mathrm{~A}, \mathrm{~B})$. Similarly, results of colony formation assay also demonstrated that the colon formation rates were obviously lower in REG $\gamma$ silenced group than that in control group and gradually decreased in REG $\gamma$ expression-dependent manner (Figure 3C, D). In addition, data from EdU assay also revealed that REG $\gamma$ depletion significantly decreased the number of cells in proliferative period (Figure 3E, F). Taken together, we found that REG $\gamma$ knockdown inhibits OS cells proliferation.

4. REG $\gamma$ knockdown induces apoptosis and cell cycle arrest in OS cell lines

To elucidate the underlying mechanism of silencing REG $\gamma$ inhibiting OS proliferation, we performed flow cytometry experiments and western blot analysis. We observed that the percentage of OS cells was increased in G0/G1 phase while decreased in S and G2/M phase following REG $\gamma$ downregulation in flow cytometry (Figure 4A, B, C, D). Furthermore, we also measured the effect of REG $\gamma$ knockdown on OS cells apoptosis and found that the reduction of REG $\gamma$ dramatically increased OS cells apoptotic rate determined by flow cytometry $(p<0.05)$ 
210 (Figure 4E, F, G, H). In addition, we quantified the apoptosis and cell cycle related genes in OS

211 cells after REG $\gamma$ knockdown at protein levels. We found that the expression of bcl-2 was

212 downregulated after transfected with siRNA-REG $\gamma$, while caspase-3 and cleaved caspase-3 were

213 upregulated (Figure 4I, J). Furthermore, cell cycle related genes were also significantly altered

214 by siRNA- REG $\gamma$. Compared with Si-NC group, higher expression of $\mathrm{p} 21$ and lower expression

215 of cyclinD1 were observed in siRNA-REG $\gamma$ groups (Figure 4C, D).

\section{Discussion:}

217 In this study, we investigated the potential role of REG $\gamma$ in human osteosarcoma. We revealed

218 that REG $\gamma$ was significantly overexpressed in human osteosarcoma tissues and cell lines for the

219 first time. Knockdown of REG $\gamma$ inhibited osteosarcoma cells proliferation, prompted cells

220 apoptosis and cell cycle arrest, indicating that targeting REG $\gamma$ could be an alternative for human

221 osteosarcoma therapy in the future.

222 Murata et al found that REG $\gamma$-deficient mice display growth retardation compared with wild

223 type mice. Meanwhile, previous studies reported that REG $\gamma$ was strongly implicated in many

224 kinds of cancer cells proliferation(Chen et al. 2018; Li et al. 2015a; Zhang et al. 2012). Our

225 results are consistent with these data, indicating that REG $\gamma$ plays a critical role in osteosarcoma

226 proliferation. To seek further the underlying mechanism that REG $\gamma$ prompting human

227 osteosarcoma proliferation, we used RNA interference to reduce REG $\gamma$ expression in MG-63

228 and SaoS-2 human osteosarcoma cell lines, then performed cell-cycle profile and apoptosis

229 analysis by flow cytometry and measured the cell cycle and apoptosis related proteins level.

230 Knockdown of REG $\gamma$ lead more cells to be arrested at the G0/G1 phase in our experiments, 
231 that was parallel with other independent studies on prostate cancer cells(Chen et al. 2017) and

232 renal carcinoma cells(Chen et al. 2018). However, another study also demonstrated that REG $\gamma$

233 downregulation resulted in cell cycle arrest at the G2/M phase in HeLa cells(Chen et al. 2007).

234 Therefore, we speculated that REG $\gamma$ depletion lead to cell cycle arrest by different mechanisms

235 in different cell types. Moreover, apoptotic percent of osteosarcoma cells transfected with Si-

236 REG $\gamma$ was higher than that in Si-NC group. Meanwhile, we found that proapoptotic protein

237 (caspase-3) level was significantly increased, while antiapoptotic protein (bcl-2) was decreased

238 following REG $\gamma$ knockdown. Present results were supported by previous researches(Chen et al.

239 2018; Moncsek et al. 2015).

240 Additionally, we discovered that proteins regulating cell cycle also were altered by siRNA

241 interference of REG $\gamma$. CyclinD1, known as a regulator of cyclin-dependent kinases, is

242 indispensable in transition from G0/G1 phase to S phase and has been reported to serve as an

243 oncogene in several types of cancers, including colorectal cancer(Wei et al. 2019), breast

244 cancer(Hosseini et al. 2019) and osteosarcoma(Li et al. 2017). Therefore, we investigated the

245 cyclinD1 expression and disclosed that level of cyclinD1 protein was gradually downregulated in

246 Si-REG $\gamma$ groups. Consistent with the phenomenon that more cells transfected with $\mathrm{Si}-\mathrm{REG} \gamma$

247 were arrested at the G0/G1 phase than cells transfected with Si-NC. A previous study also has

248 shown that the depletion of REG $\gamma$ leads to a striking decrease in cyclinD1 levels in prostate

249 cancer cells. Taking all these results into account, we demonstrated that cyclinD1 may play an

250 important part in the REG $\gamma$-related control of cell cycle progression in osteosarcoma. In

251 addition, p21 serves as a broad-spectrum cyclin-dependent kinases inhibitor and takes part in 
252 regulating the cell cycle in many types of cells(Weinberg \& Denning 2016). However, there has

253 been significant debate as to whether the REG $\gamma$ depletion increases p 21 protein expression level.

254 Previous studies revealed that the reduction of REG $\gamma$ lead to a markedly increase in p21 in TPC

255 cells, but only had a relatively slight effect on p21 levels in MCF-7 cells and even had no effect

256 on p21 expression in HepG2 and 3T3-L1 cells(Chen et al. 2007), indicating a cell type-specific

257 effect of REG $\gamma$ depletion on p21 protein expression. Given distinct effect of REG $\gamma$ depletion on

258 different kinds of cells, we measured the p21 level in cells after transfection. Interestingly, we

259 conformed for the first time that REG $\gamma$ knockdown significantly increases the level of the p21

260 protein in osteosarcoma cell lines in the present study.

261 REG $\gamma$ can regulate expression of multiple proteins, the specific mechanisms by which it plays

262 its pivotal roles have been preliminarily elucidated in previous research. The cell cycle inhibitor

p21 has also been identified to be a REG $\gamma$-proteasome target (Chen et al 2007; Li et al 2007). It

was reported that the ubiquitin-independent REG $\gamma$-proteasome pathway is responsible for the

degradation of p21 in vivo and in vitro. P53, tumor suppressor protein, have the ability to

stimulate apoptosis and cell cycle arrest in the event of DNA damage and strongly suppress

oncogenesis (Sharpless, N. E. et al 2002). REG $\gamma$ has been found to be a cofactor that assists the

interaction between 53 and MDM2 by specifically binding to both proteins and promoting the

ubiquitin-dependent proteasomal degradation of p53 (Zhang et al 2008). In addition, recent

studies on post-translational modification of REG $\gamma$ revealed an important regulatory mechanism

for its activities. Researches have showed that REG $\gamma$ can be phosphorylated in vitro and further 
273

274

275

276

277

278

279

280

281

282

283

284

285

286

287

288

289

290

291

292

293

MEKK3, a mitogen-activated protein kinase (MAPK) kinase, activates JNK and p38 MAPKs, directly binds with and phosphorylates REG $\gamma$ (Hagemann, C et al 2003). Another MAPK

kinase, B-RAF, which activates ERK1/2 MAPK, has also been shown to interact with REG $\gamma$ and

phosphorylate REG $\gamma$ in vitro (Hagemann, C et al 2003; Hagemann, C et al 1999).

In this study, the underlying mechanisms of REG $\gamma$ in promoting OS proliferation were not investigated in depth. Nevertheless, current study sheds light on a new perspective for OS treatment. Further researches assessing the deep contribution of REG $\gamma$ in OS are warranted.

\section{Conclusions:}

We revealed for the first that REG $\gamma$ was significantly overexpressed in human osteosarcoma tissues and cell lines for the first time. Knockdown of REG $\gamma$ inhibited osteosarcoma cells proliferation, prompted cells apoptosis and cell cycle arrest, we speculate that the REG $\gamma$ controlled proliferation of osteosarcoma cells is probably due to the role of REG $\gamma$ in regulating the cell-cycle and apoptosis relevant proteins, but the specific mechanism is still unclear. Thus, further study is needed to comprehensively understand the importance of REG $\gamma$ in the development and progression of osteosarcoma.

\section{Acknowledgements:}

None.

\section{Disclosure of conflict of interest:}

The authors declare no conflict of interest.

\section{Funding Sources:}

This study was supported by the Natural Science Foundation of Heilongjiang Province, China 
294

295

296

297

298

299

300

301

302

303

304

305

306

307

308

309

310

311

312

313

314

315

316

317

318

319

320

321

322

323

324

325

326

327

328

329

330

331

332

(Grant NO. QC2016102 and H2016002).

\section{References:}

Botter SM, Neri D, and Fuchs B. 2014. Recent advances in osteosarcoma. Curr Opin Pharmacol 16:15-23. 10.1016/j.coph.2014.02.002

Chen S, Wang Q, Wang L, Chen H, Gao X, Gong D, Ma J, Kubra S, Yao X, Li X, Li L, Zhai W, and Zheng J. 2018. REGgamma deficiency suppresses tumor progression via stabilizing CK1 epsilon in renal cell carcinoma. Cell Death Dis 9:627. 10.1038/s41419-018-0646-2

Chen X, Barton LF, Chi Y, Clurman BE, and Roberts JM. 2007. Ubiquitin-independent degradation of cell-cycle inhibitors by the REGgamma proteasome. Mol Cell 26:843-852. 10.1016/j.molcel.2007.05.022

Hagemann, C. and Rapp, U. R. 1999. Isotype-specific functions of Raf kinases. Exp. Cell Res 253:34-46. 10.1006/excr.1999.4689

Hagemann, C., Patel, R. and Blank, J. L. 2003. MEKK3 interacts with the PA28 gamma regulatory subunit of the proteasome. Biochem. J 373:71-79. 10.1042/BJ20021758

Hosseini S, Chamani J, Hadipanah MR, Ebadpour N, Hojjati AS, Mohammadzadeh MH, and Rahimi HR. 2019. Nano-curcumin's suppression of breast cancer cells (MCF7) through the inhibition of cyclinD1 expression. Breast cancer (Dove Medical Press) 11:137-142. 10.2147/BCTT.S195800

Kempf-Bielack B, Bielack SS, Jurgens H, Branscheid D, Berdel WE, Exner GU, Gobel U, Helmke K, Jundt G, Kabisch H, Kevric M, Klingebiel T, Kotz R, Maas R, Schwarz R, Semik M, Treuner J, Zoubek A, and Winkler K. 2005. Osteosarcoma relapse after combined modality therapy: an analysis of unselected patients in the Cooperative Osteosarcoma Study Group (COSS). J Clin Oncol 23:559-568. 10.1200/JCO.2005.04.063

Laura Zannini,Daniele Lecis, Giacomo Buscemi, Luigi Carlessi, Patrizia Gasparini,Enrico Fontanella,Sofia Lisanti, Lance Barton and Domenico Delia, 2008. REG $\gamma$ proteasome activator is involved in the maintenance of chromosomal stability. Cell Cycle 7:504-512. 10.4161/cc.7.4.5355

Li L, Dang Y, Zhang J, Yan W, Zhai W, Chen H, Li K, Tong L, Gao X, Amjad A, Ji L, Jing T, Jiang Z, Shi K, Yao L, Song D, Liu T, Yang X, Yang C, Cai X, Xu W, Huang Q, He J, Liu J, Chen T, Moses RE, Fu J, Xiao J, and Li X. 2015a. REGgamma is critical for skin carcinogenesis by modulating the Wnt/beta-catenin pathway. Nat Commun 6:6875. 10.1038/ncomms7875

Li L, Zhao D, Wei H, Yao L, Dang Y, Amjad A, Xu J, Liu J, Guo L, Li D, Li Z, Zuo D, Zhang Y, Liu J, Huang S, Jia C, Wang L, Wang Y, Xie Y, Luo J, Zhang B, Luo H, Donehower LA, Moses RE, Xiao J, O'Malley BW, and Li X. 2013. REGgamma deficiency promotes premature aging via the casein kinase 1 pathway. Proc Natl Acad Sci U S A 110:11005-11010. 10.1073/pnas.1308497110

Li S, Cheng D, Zhu B, and Yang Q. 2017. The Overexpression of CARM1 Promotes Human Osteosarcoma Cell Proliferation through the pGSK3 $\beta / \beta$-Catenin/cyclinD1 Signaling Pathway. International journal of biological sciences 13:976-984. 10.7150/ijbs.19191

Li S, Jiang C, Pan J, Wang X, Jin J, Zhao L, Pan W, Liao G, Cai X, Li X, Xiao J, Jiang J, and Wang P. 2015 b. Regulation of c-Myc protein stability by proteasome activator REGgamma. Cell Death Differ 22:10001011. 10.1038/cdd.2014.188

Li X, Lonard DM, Jung SY, Malovannaya A, Feng Q, Qin J, Tsai SY, Tsai MJ, and O'Malley BW. 2006. The SRC- 
333

334

335

336

337

338

339

340

341

342

343

344

345

346

347

348

349

350

351

352

353

354

355

356

357

358

359

360

361

362

363

364

365

366

367

368

369

370

3/AIB1 coactivator is degraded in a ubiquitin- and ATP-independent manner by the REGgamma proteasome. Cell 124:381-392. 10.1016/j.cell.2005.11.037

Li, X, Amazit, L, Long,W. Lonard, D. M, Monaco, J. J. and O_Malley, B. W. 2007. Ubiquitin- and ATPindependent proteolytic turnover of p21 by the REGgamma-proteasome pathway. Mol. Cell 26: 831-842. 10.1016/j.molcel.2007.05.028

Ma Chu-Ping, Patricia J. Willy, Clive A. Slaughterjll, and George N. DeMartino. 1993. PA28, an Activator of the 20 S Proteasome, Is Inactivated by Proteolytic Modification at Its Carboxyl Terminus. J Bio chem 268:2251422519.

Marko TA, Diessner BJ, and Spector LG. 2016. Prevalence of Metastasis at Diagnosis of Osteosarcoma: An International Comparison. Pediatric blood \& cancer 63:1006-1011. 10.1002/pbc.25963

Moncsek A, Gruner M, Meyer H, Lehmann A, Kloetzel PM, and Stohwasser R. 2015. Evidence for anti-apoptotic roles of proteasome activator 28gamma via inhibiting caspase activity. Apoptosis 20:1211-1228. 10.1007/s10495-015-1149-6

Siegel RL, Miller KD, and Jemal A. 2016. Cancer statistics, 2016. CA Cancer J Clin 66:7-30. 10.3322/caac.21332

Shaojun Chen, Longsheng Wang, Chen Xu, Hui Chen, Bo Peng, Yunfei Xu, Xudong Yao, Lei Li and Junhua Zheng. 2017. Knockdown of REG $\gamma$ inhibits proliferation by inducing apoptosis and cell cycle arrest in prostate cancer. Am J Transl Res 9:3787-3795.

Sharpless, N. E. and DePinho, R. A. 2002. p53: good cop/bad cop. Cell 110:9-12. 10.1016/S0092 8674(02)00818-8

Wang H, Bao W, Jiang F, Che Q, Chen Z, Wang F, Tong H, Dai C, He X, Liao Y, Liu B, Sun J, and Wan X. 2015. Mutant p53 (p53-R248Q) functions as an oncogene in promoting endometrial cancer by up-regulating REGgamma. Cancer Lett 360:269-279. 10.1016/j.canlet.2015.02.028

Wei Y, Huang C, Wu H, and Huang J. 2019. Estrogen Receptor Beta (ER $\beta$ ) Mediated-CyclinD1 Degradation via Autophagy Plays an Anti-Proliferation Role in Colon Cells. International journal of biological sciences 15:942-952. 10.7150/ijbs.30930

Weinberg WC, and Denning MF. 2016. p21WAF1 Control of Epithelial Cell Cycle and Cell Fate. Critical Reviews in Oral Biology \& Medicine 13:453-464. 10.1177/154411130201300603

Yi Z, Yang D, Liao X, Guo F, Wang Y, and Wang X. 2017. PSME3 induces epithelial-mesenchymal transition with inducing the expression of CSC markers and immunosuppression in breast cancer. Exp Cell Res 358:87-93. 10.1016/j.yexcr.2017.05.017

Zhang M, Gan L, and Ren GS. 2012. REG $\gamma$ is a strong candidate for the regulation of cell cycle, proliferation and the invasion by poorly differentiated thyroid carcinoma cells. Brazilian Journal of Medical and Biological Research 45:459-465. 10.1590/s0100-879x2012007500035

Zhang, Z. and Zhang, R. 2008. Proteasome activator PA28 gamma regulates p53 by enhancing its MDM2-mediated degradation. EMBO J 27:852-864. 10.1038/emboj.2008.25

Figure legends:

Figure 1. REG $\gamma$ expression is upregulated in OS. (A, B, C, D) Expression of REG $\gamma$ in OS tissues (T) and adjacent normal tissues (AT) as detected by IHC (A, B), WB (C) and qRT-PCR

(D). In the Figure 1A, B, the brown represents the expression of REG $\gamma$ in OS tissues and AT.

Peer) reviewing PDF | (2019:11:42765:2:0:CHECK 17 Mar 2020) 
371 Pictures on the left and on the right are magnified 50 times and 100 times, respectively. (E, F)

372 Expression of REG $\gamma$ in two OS cell lines (MG-63 and SaoS-2) and a normal osteoblast cell line

373 (hFOB1.19), as detected by WB (E)and qRT-PCR. (G, H, I). REG $\gamma$ expression (median

expression intensity) in sarcoma tissues and adjacent normal tissues derived from the Oncomine database (https://www.oncomine.org/). ${ }^{* * \mathrm{P}}<0.01,{ }^{*} \mathrm{P}<0.05$.

Figure 2. Si-REG $\gamma$ reduce the expression of REG $\gamma$ at mRNA $(A, B)$ and protein level $(C, D)$ in OS cells. Compared to Si-NC, Si-REG $\gamma-1$ and Si-REG $\gamma$-2 inhibit more than 50 percent of REG $\gamma$ expression and Si- REG $\gamma-3$ inhibit less than 50 percent of REG $\gamma$ expression at mRNA level $(\mathrm{A}, \mathrm{B})$ and protein level $(\mathrm{C}, \mathrm{D})$. Data are shown as the mean $\pm \mathrm{SD} .{ }^{*} \mathrm{P}<0.05$.

Figure 3. REG $\gamma$ depletion suppresses OS cell progression in vitro. (A, B) Effect of Si-REG- $\gamma-1$ and Si-REG- $\gamma-2$ on OS cell growth as determined by CCK-8 assay. (C, D) Representative OS cell colony formation images after transfection of Si-REG $\gamma$ versus Si-NC. (E, F) Representative images of the EdU incorporation assay after transfection of Si-REG $\gamma$ compared to after transfection of Si-NC in Mg-63 (E) and in SaoS-2 (F). Data are shown as the mean \pm SD. *P $<0.05$.

Figure 4. REG $\gamma$ deficiency induce apoptosis and cell cycle arrest and alters multiple cell apoptosis and cell cycle related proteins in MG-63 and SaoS-2. Representative flow cytometry analysis of the cell cycle distribution of MG-63 and SaoS-2 cells transfected with Si-REG $\gamma$ and

Si-NC. (A, B, C, D) Apoptosis rate of MG-63 and SaoS-2 cells after transfection with Si-REG $\gamma$ and Si-NC, as determined by flow cytometry (E, F, G, H). Si- REG $\gamma$ alters apoptosis and cell cycle related genes at protein levels (I, J). Data are shown as the mean $\pm \mathrm{SD}, * \mathrm{P}<0.05$. 


\title{
Figure 1
}

\section{REG $\mathrm{Y}$ expression isupregulated in OS.}

\begin{abstract}
$(A, B, C, D)$ Expression of REG $\gamma$ in OS tissues $(T)$ and adjacent normal tissues (AT) as detected by IHC $(A, B)$, WB (C) and qRT-PCR (D). In the Figure 1A, B, the brown represents the expression of REG $\gamma$ in OS tissues and AT. Pictures on the left and on the right are magnified 50 times and 100 times, respectively. ( $E, F)$ Expression of REG $y$ in two OS cell lines (MG-63 and SaoS-2) and a normal osteoblast cell line (hFOB1.19), as detected by WB (E)and qRT-PCR. ( G, H, I). REG Y expression (median expression intensity) in sarcoma tissues and adjacent normal tissues derived from the Oncomine database (https://www.oncomine.org/). **P $<0.01, * \mathrm{P}<0.05$.
\end{abstract}


A

Patient 1
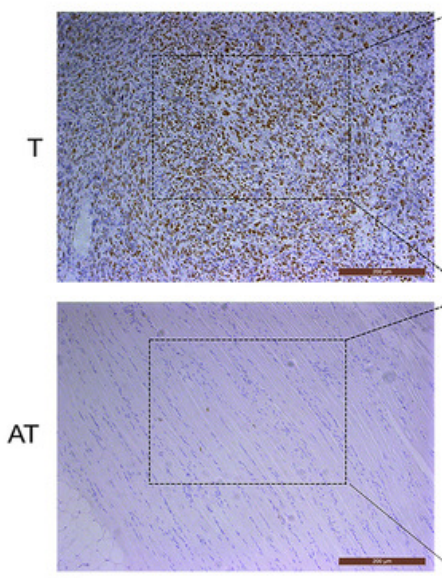

C
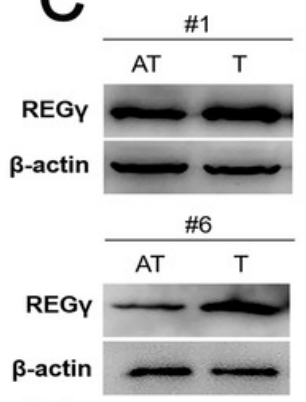

E
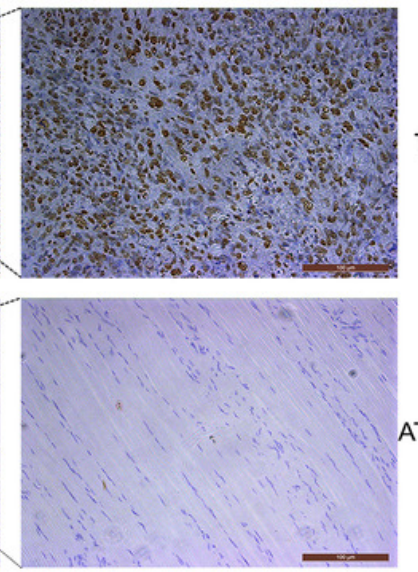

B

Patient 2
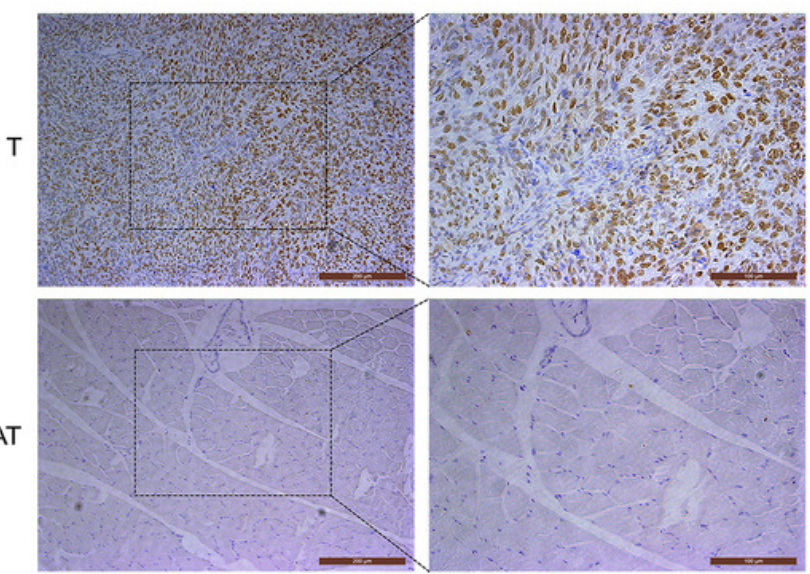

D

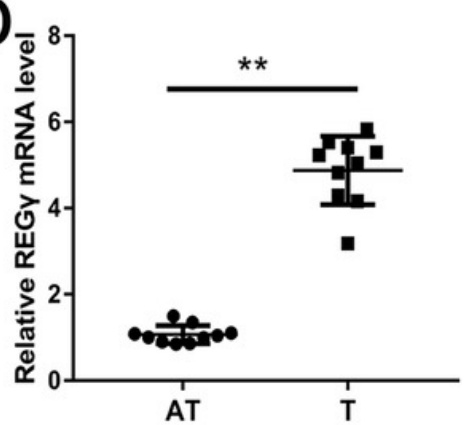

$\mathbf{F}$
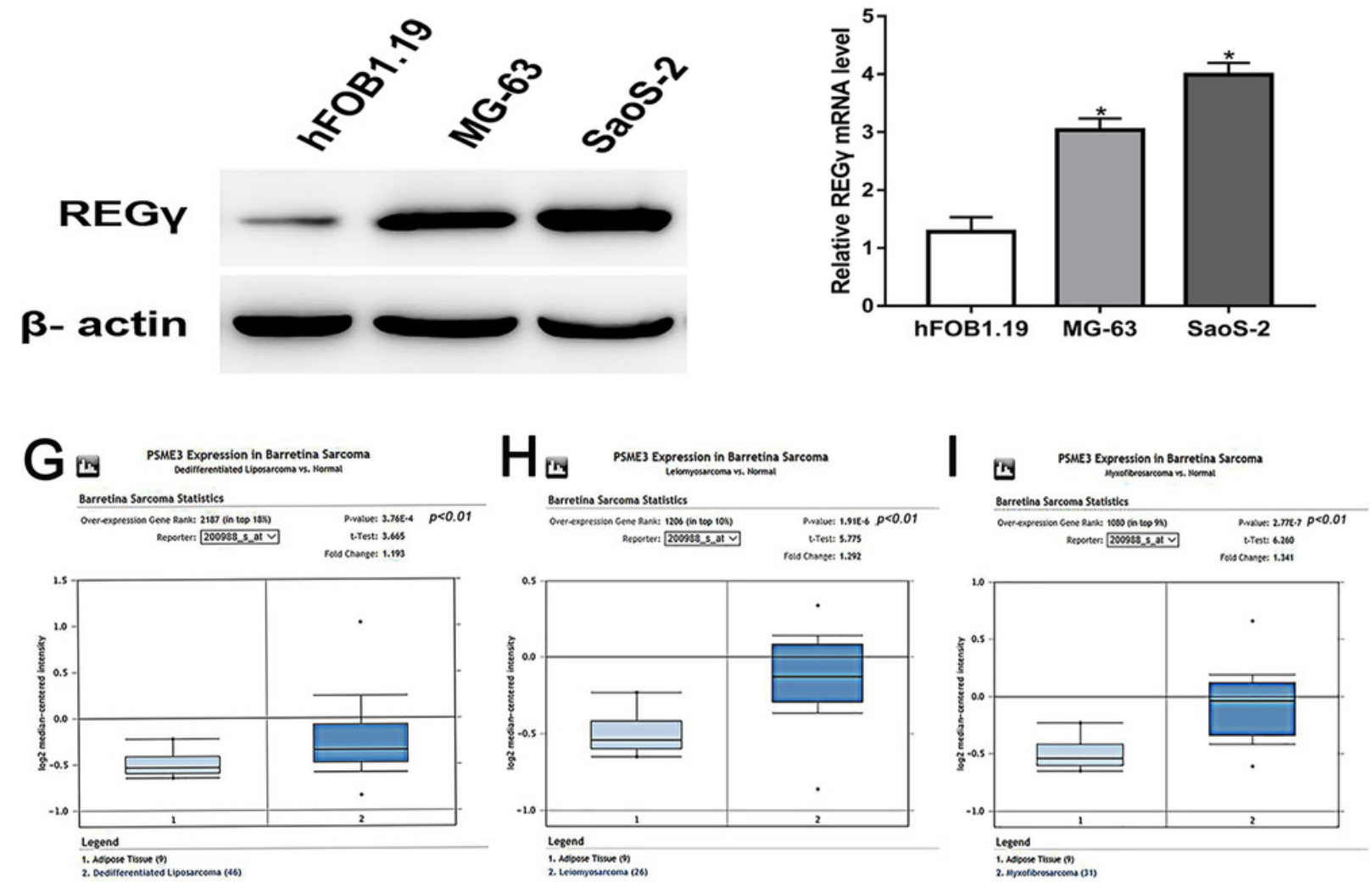
Figure 2

\section{Si- REG $\gamma$ reduce the expressionof REG $Y$.}

Compared to Si-NC, Si- REG $\gamma$-1 and Si- REG $\gamma$-2 inhibit more than 50 percent of REG $\gamma$ expression and SiREG $\gamma-3$ inhibit less than 50 percent of REG $\gamma$ expression at mRNA level (A, B) and protein level (C, D). Data are shown as the mean $\pm \mathrm{SD}$. $* \mathrm{P}<0.05$. 
A

MG-63

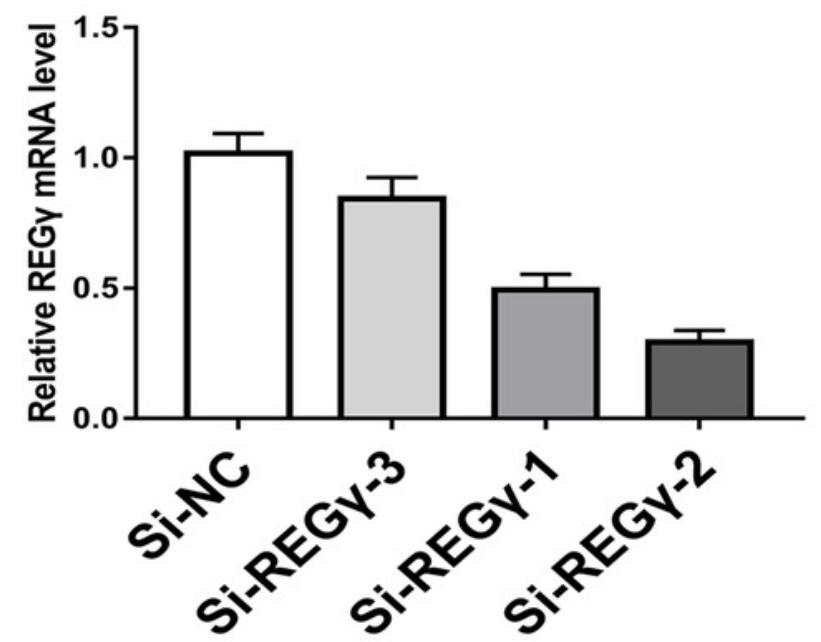

C

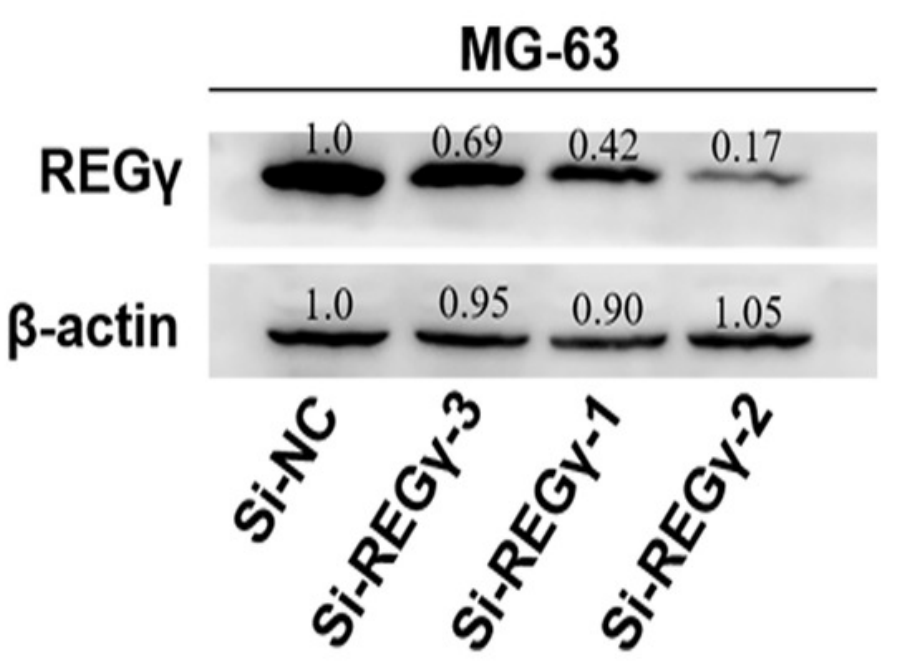

B

SaoS-2

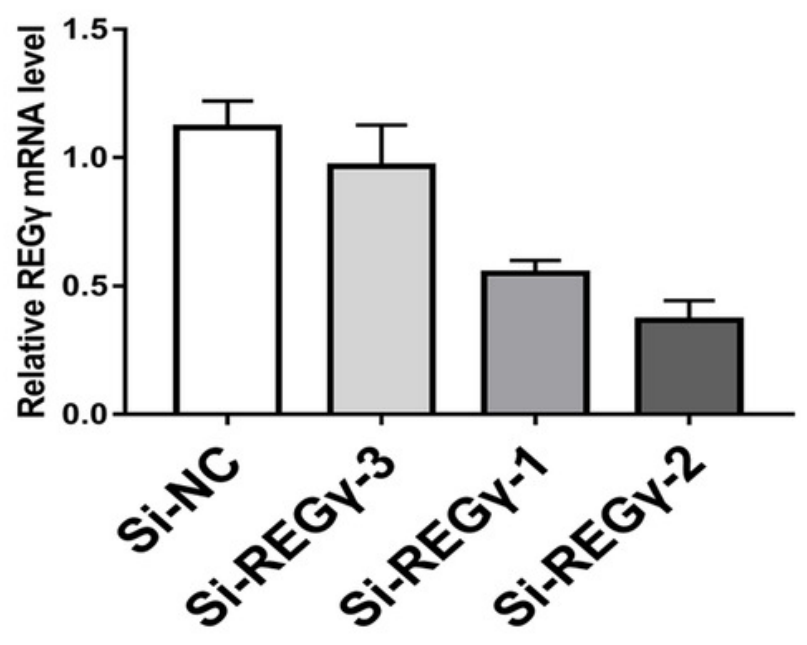

D

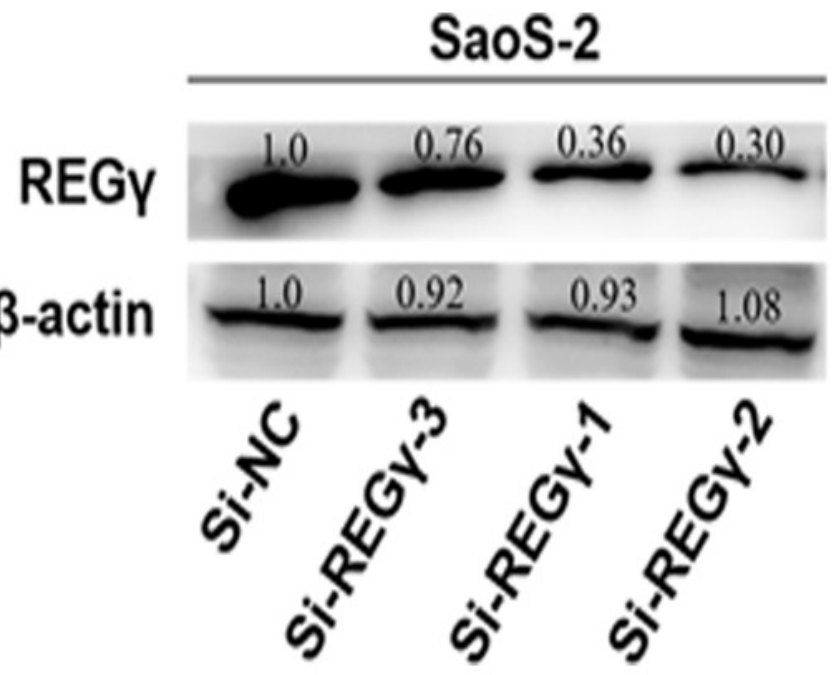




\section{Figure 3}

REG Y depletion suppresses OS cell progression invitro.

REG $\gamma$ depletion suppresses OS cell progression in vitro. (A, B) Effect of Si-REG- $\gamma-1$ and Si-REG- $\gamma-2$ on OS cell growth as determined by CCK-8 assay. (C, D) Representative OS cell colony formation images after transfection of Si-REG $y$ versus Si-NC. (E, F) Representative images of the EdU incorporation assay after transfection of Si-REG y compared to after transfection of Si-NC in Mg-63 (E) and in SaoS-2 (F). Data are shown as the mean $\pm \mathrm{SD}$. $* P<0.05$. 
A

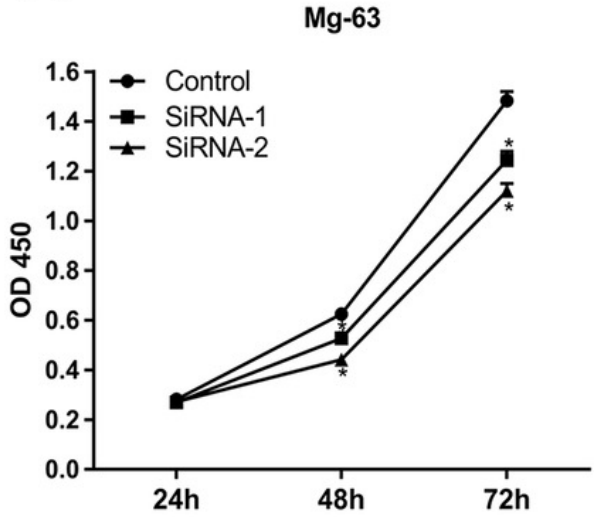

B

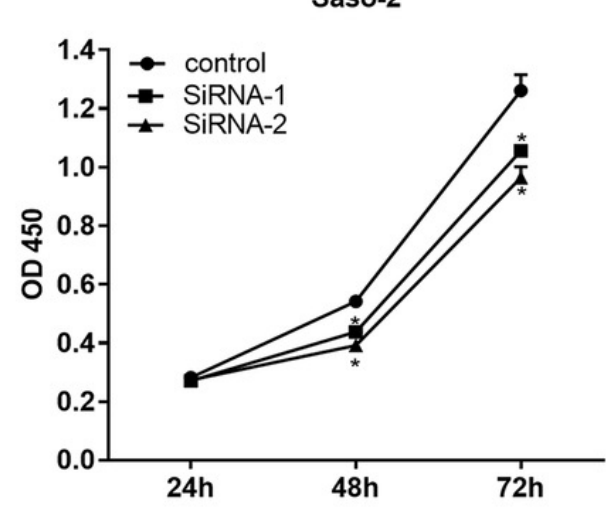

C
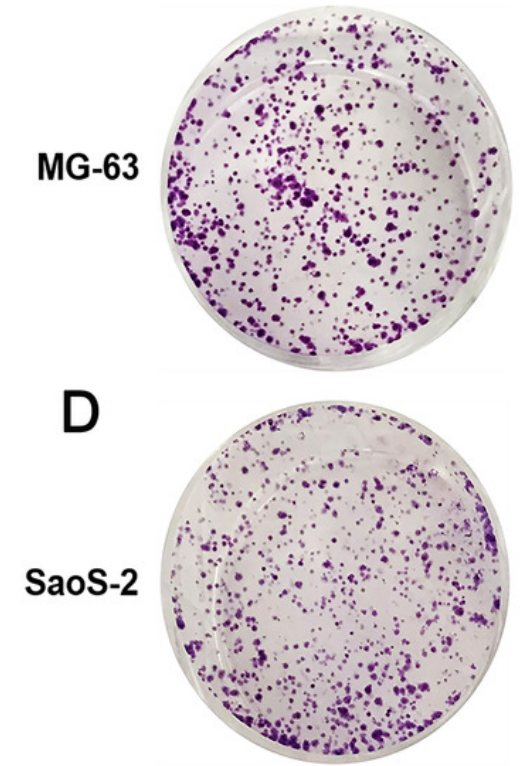

E

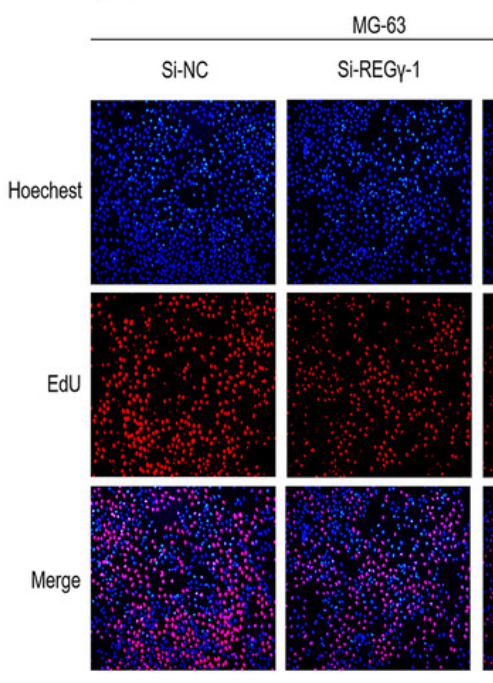

Si-REGy-1
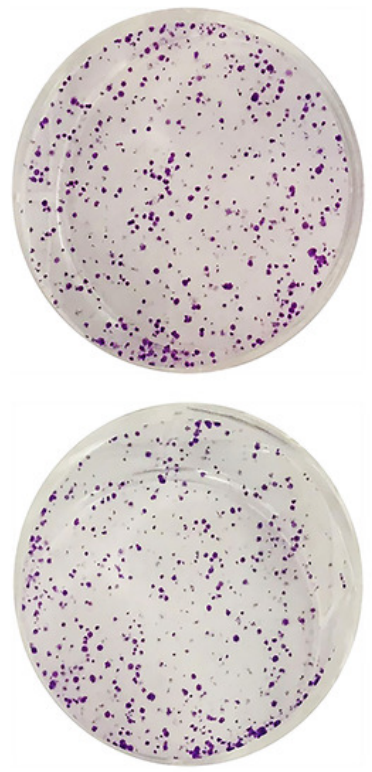

F
Si-REGy-2
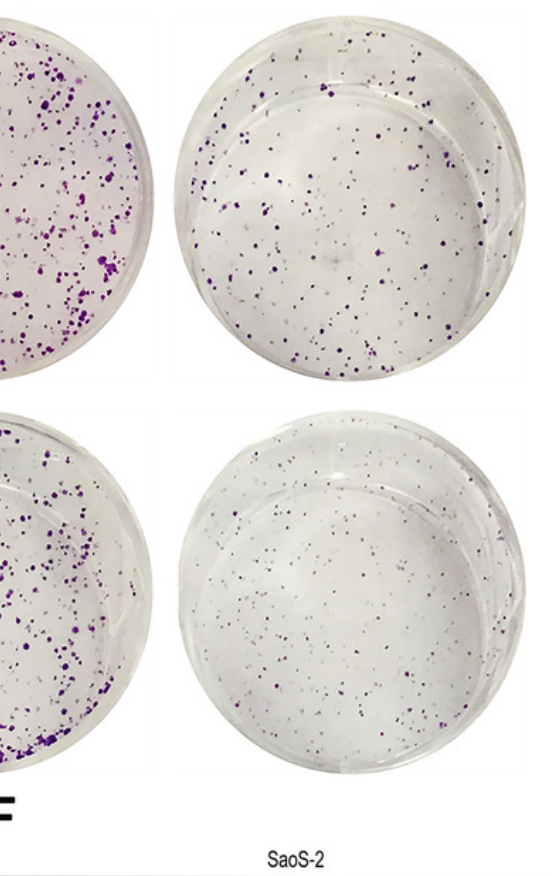
Saos-2 Si-REGY-2
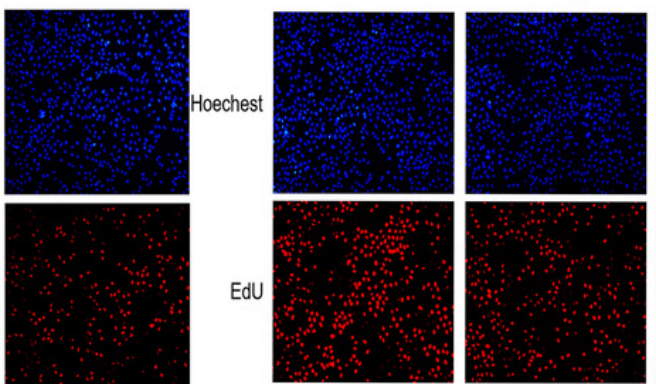

Si-REGy-2

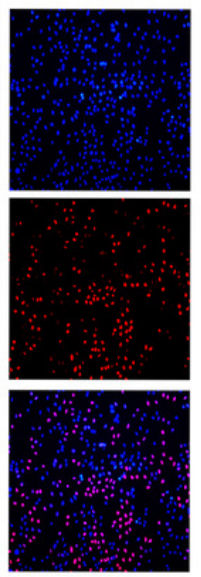




\section{Figure 4}

REG $\mathrm{Y}$ deficiency induce apoptosis and cell cycle arrest andalters multiple cell apoptosis and cell cycle related proteins in MG-63 andSaoS-2.

REG Y deficiency induce apoptosis and cell cycle arrest and alters multiple cell apoptosis and cell cycle related proteins in MG-63 and SaoS-2. Representative fl ow cytometry analysis of the cell cycle distribution of MG-63 and SaoS-2 cells transfected with Si- REG $\gamma$ and Si-NC. (A, B, C, D) Apoptosis rate of MG-63 and SaoS-2 cells after transfection with Si-REG $\gamma$ and Si-NC, as determined by fl ow cytometry $(E, F, G, H)$. SiREG $Y$ alters apoptosis and cell cycle related genes at protein levels (I, J). Data are shown as the mean \pm $\mathrm{SD}, * P<0.05$. 
A

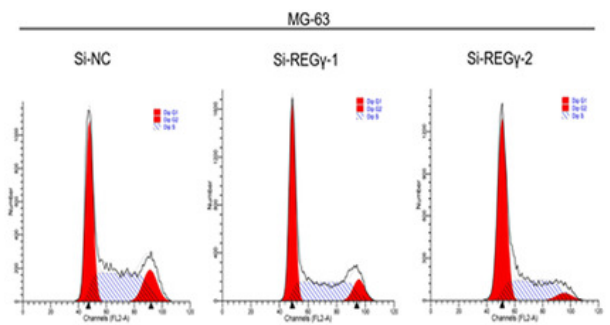

C

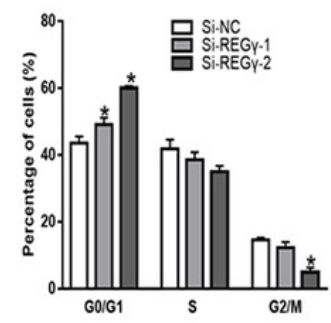

E
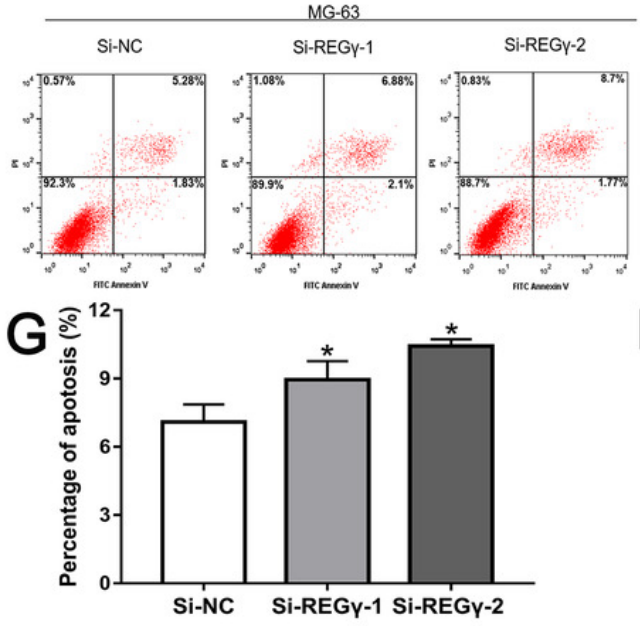

I

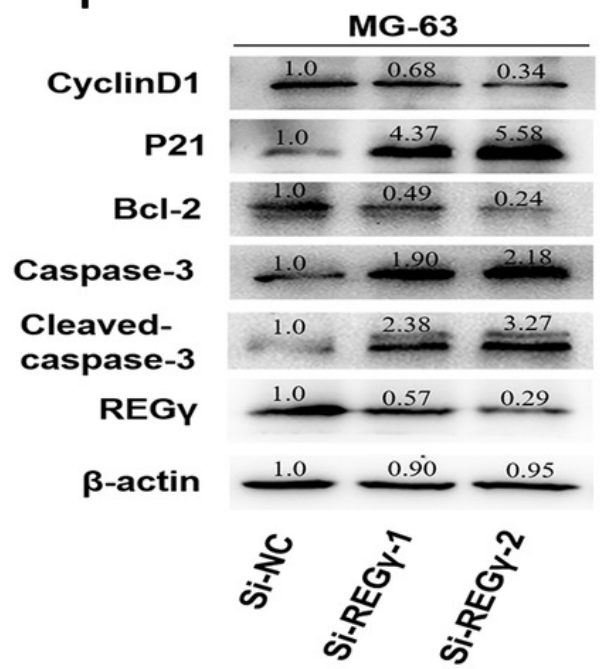

B

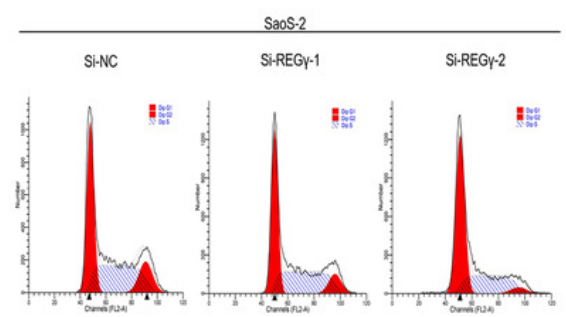

D

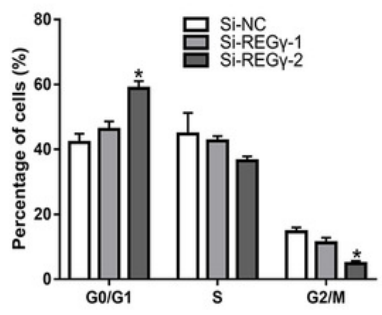

F
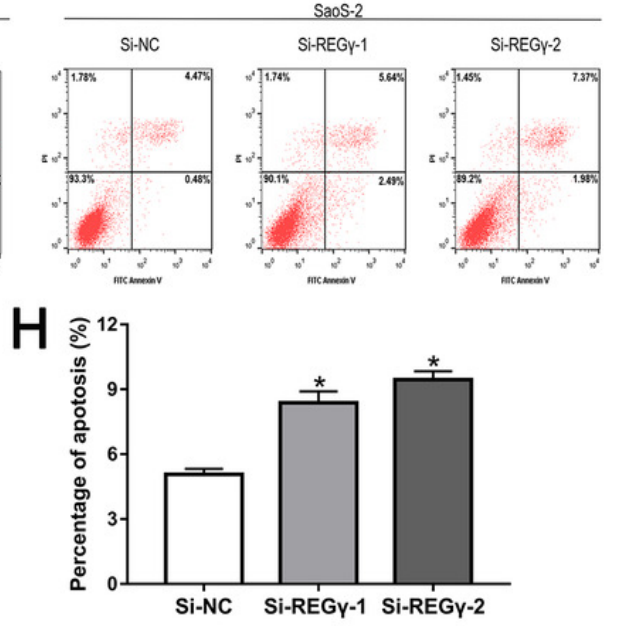

J

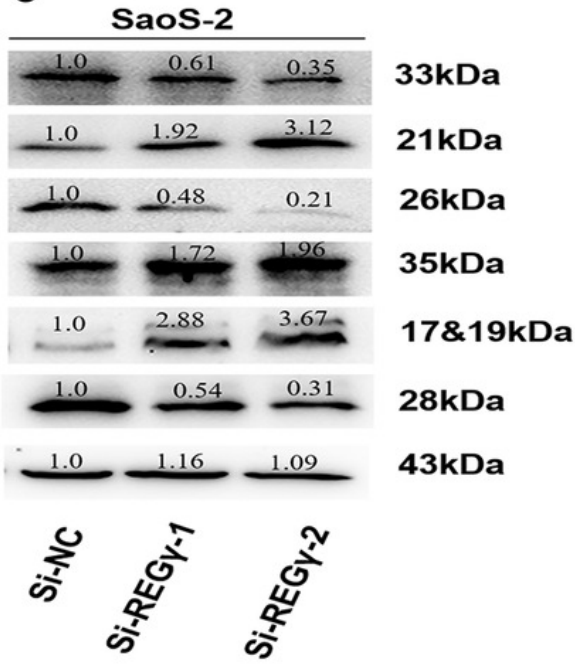




\section{Table 1 (on next page)}

Sequences ofSi-RNA and primers. 
1 Table 1: Sequences of Si-RNA and primers:

\begin{tabular}{|c|c|}
\hline Name: & Sequence: \\
\hline \multirow[t]{2}{*}{ Si-negative control } & Sense:5'-UUCUCCGAACGUGUCACGUTT-3' \\
\hline & Antisense: 5'-ACGUGACACGUUCGGAGAATT-3' \\
\hline \multirow[t]{2}{*}{ Si-REG $\gamma-1$} & Sense: 5'-GCAGAAGACUUGGUGGCAATT-3' \\
\hline & Antisense:5'-UUGCCACCAAGUCUUCUGCTT \\
\hline \multirow[t]{2}{*}{ Si-REG $\gamma-2$} & Sense: 5'-CCAAGGAACCAAGGUGUUUTT-3' \\
\hline & Antisense:5'-AAACACCUUGGUUCCUUGGTT-3' \\
\hline \multirow[t]{2}{*}{ Si-REG $\gamma-3$} & Sense: 5'-GGAUAGAAGAUGGAAACAATT-3' \\
\hline & Antisense: 5'-UUGUUUCCAUCUUCUAUCCTT-3' \\
\hline \multirow[t]{2}{*}{ GAPDH: } & Sense:5'-CСАСТССТССАССТTTGAC -3' \\
\hline & Antisense: 5'-ACCCTGTTGCTGTAGCCA -3' \\
\hline \multirow[t]{2}{*}{ REG $\gamma$} & Sense: 5'-CTCCTGATACTGTAGCCTCTTGG -3' \\
\hline & Antisense: 5'-AGCATCTGGACCTCACACTTG -3' \\
\hline
\end{tabular}

2 
Table 2 (on next page)

Clinical characteristics of osteosarcomapatients. 
1 Table 1 clinical characteristics of osteosarcoma patients:

\begin{tabular}{|c|c|c|c|c|c|c|}
\hline & Age & Gender & Location & Size (cm) & $\begin{array}{l}\text { Tumor } \\
\text { Stage }\end{array}$ & Metastasis \\
\hline Patient 1 & 15 & $\mathrm{~F}$ & $\begin{array}{l}\text { Proximal } \\
\text { Fibula }\end{array}$ & 3.5 & IIA & No \\
\hline Patient 2 & 18 & $\mathrm{M}$ & $\begin{array}{l}\text { Proximal } \\
\text { Tibia }\end{array}$ & 2.0 & IB & No \\
\hline Patient 3 & 14 & $\mathrm{~F}$ & $\begin{array}{l}\text { Distal } \\
\text { Femur }\end{array}$ & 6.8 & IIIA & Yes \\
\hline Patient 4 & 13 & $\mathrm{~F}$ & $\begin{array}{l}\text { Distal } \\
\text { Femur }\end{array}$ & 5.0 & IIB & No \\
\hline Patient 5 & 14 & $\mathrm{M}$ & $\begin{array}{l}\text { Proximal } \\
\text { Tibia }\end{array}$ & 5.5 & IIIA & Yes \\
\hline Patient 6 & 26 & $\mathrm{M}$ & $\begin{array}{l}\text { Proximal } \\
\text { Tibia }\end{array}$ & 2.5 & IIA & No \\
\hline Patient 7 & 16 & $\mathrm{~F}$ & $\begin{array}{l}\text { Distal } \\
\text { Femur }\end{array}$ & 3.4 & IIA & No \\
\hline Patient 8 & 23 & $\mathrm{M}$ & $\begin{array}{l}\text { Proximal } \\
\text { Tibia }\end{array}$ & 4.2 & IIB & No \\
\hline Patient 9 & 11 & $\mathrm{M}$ & $\begin{array}{l}\text { Proximal } \\
\text { Tibia }\end{array}$ & 6.5 & IIIB & Yes \\
\hline Patient 10 & 20 & $\mathrm{M}$ & $\begin{array}{l}\text { Distal } \\
\text { Femur }\end{array}$ & 3.7 & IIB & No \\
\hline
\end{tabular}

2 